\title{
Biophysical Studies of Cancer Cells' Traverse- Vessel Behaviors Under Different Pressures Revealed Cells' Motion State Transition
}

\section{Xiao LI}

Peking University

Jialin SHI

Peking University

\section{Ziqing GAO}

Peking University

Jian XU

Peking University

Shujing WANG

Peking University

Qi Ouyang

Peking University

Chunxiong Luo ( $\nabla$ pkuluocx@pku.edu.cn )

Peking University

\section{Research Article}

Keywords: capillary sized vessel, cancer cell, biophysical, high-throughput, microfluidics.

Posted Date: August 27th, 2021

DOl: https://doi.org/10.21203/rs.3.rs-841772/v1

License: (c) (1) This work is licensed under a Creative Commons Attribution 4.0 International License. Read Full License 


\section{Abstract}

Circulating tumor cells (CTCs) survive in the bloodstream, seed, and invade to foster tumor metastasis. The arrest of CTCs is favored by permissive flow forces and geometrical constraints. Through the use of high-throughput microfluidic devices designed to mimic capillary-sized vessels, we applied different pressure differences to cancer cells and recorded cell traverse-vessel behaviors. Our results showed that cancer cells would transform from Newtonian droplet state to adhesion/migration state when cancer cells traverse in the artificial vessels. To explain these phenomena, a modified Newtonian droplet model was also proposed. These phenomena and the modified model may reveal how the CTCs in the blood seed and invade in the vessels under suitable conditions.

\section{Introduction}

Cancer metastasis is the key cause of the cancer mortality rate [1]. Primary tumor releases circulating tumor cells (CTCs) into the circulatory and lymphatic system to colonize distant organs which causes metastasis. In order to colonize distant organs, CTCs must be able to overcome the mechanical pressures imposed by architectural constraints of tissues, squeeze through capillary-sized microvessels, and adhere/seed at a special location within their lifespan. These events in the metastatic processes involve the interplay between chemical signals and physical interactions. Chemical signals have long been studied for metastasis [2-3]. However, more researches about physical interactions need to be further deepened. When CTCs flow with blood in the blood vessels, the size and the mechanical properties of cancer cells in certain environments play a key role to squeeze through capillary-sized microvessels and adhere/seed in a special place in the cancer metastatic processes. Researches on the mechanical properties of cancer cells might help to understand the biophysical mechanisms of cancer metastasis and provide some insights for cancer therapies.

Many specialized tools have been developed to measure mechanical properties of the single cancer cell, such as atomic force microscopy (AFM), optical tweezer, magnetic twisting cytometry and micropipette. Depending on different spatiotemporal scales and different cell stages, the mechanical properties measured by these methods might vary in one to three orders of magnitude, even for the same type of cells [4-5]. As a major approach to study the mechanical properties of single cells both in the suspension stage and in adhere stage, conventional micropipette aspiration has been implemented in many fields of cell mechanics for many years [6-7]. Nevertheless, this traditional method needs skilled laboratorians to operate one cell at a time. In recent years, microfluidic-based methods for cell mechanics have been developed quite rapidly. Compared to other existing methods, microfluidic assays are high throughput and easy to implement. Integrated with time-lapse microscopes, these assays could produce highresolution data in an automated manner [8-13]. These microfluidic-based methods not only facilitated the studies of cell mechanics, but also helped to understand the biophysical process of cancer cells to transfer in the artificial blood vessel. 
But for now, most of these studies focused on the mechanical properties of single cancer cells or cell clusters responding to high mechanical stresses on timescales of one to ten seconds $[4,8,10-13]$ and researches about dynamic behaviors of cells in confinement pace emphasis too much on cell migration [16-19]. In fact, human physiological systems are intrinsically complex. For the circulatory system, normal blood pressure ranges from $3 \mathrm{mmHg}$ to $120 \mathrm{mmHg}$ [20]. Yet, the physiological pressure inside the lymph capillary is around $1 \sim 3 \mathrm{mmHg}$ [21]. During metastasis, it mostly takes hours or days for CTCs to invade and traverse through blood and lymph capillaries [22-23]. At long timescales, it is likely that the CTCs' stages might change during traveling in the vessels which influences their behavioral patterns. From this perspective, researches about the dynamic behaviors of single cells in vessels exerted low pressure might help us find the critical piece in the jigsaw puzzle.

In this work, a new microfluidic device was engineered to capture single cells into a capillary-shaped vessels array and then designed pressures were applied to these cells. In one chip, we could study the dynamic traverse-vessel behaviors of tens of single cancer cells with four designed low pressures for hours. Our results demonstrate that cancer cells could transform their state from Newtonian droplet state to adhesion/migration state over time when they were trapped in vessels. During these transformations, the apparent viscosities of cancer cells would increase as the applied pressure decreased. The critical transition of Newtonian droplet state to adhesion/migration state was theoretically analyzed and experimentally confirmed by varying the pressures and the vessel sizes.

\section{Results}

\section{Device design and test}

The design concept and a photograph of the whole microfluidic device are shown in Fig. 1A and 1B. The microfluidic device was manufactured by bonding the $\mathrm{CO}_{2}$ layer (upper) on the cell cultivation layer (bottom) using air plasma. Throughout the experiment, $5 \% \mathrm{CO}_{2}$ was pumped into the upper layer and diffused into the bottom layer. Meanwhile, the whole microfluidic system was heated up to $37^{\circ} \mathrm{C}$. These methods could maintain the vitalities of single cells trapped in microvessels.

Specifically, cell cultivation layer consists of four parallel identical microchannels with inlet, filter, trapping area and outlet as detailed in Fig.1C. The filter structures (20 $\mu \mathrm{m}$ in width) are designed to block cell clusters and large debris. And the trapping area is composed of a main square-wave shaped loop channel (40 $\mu \mathrm{m}$ in width, $20 \mu \mathrm{m}$ in height, 40 loop and $7.2 \mathrm{~mm}$ long for each loop) and repeated triplet capillarylike straight channels positioned along both sides of the axis. For microvessels, there are two sizes available in our experiments. The width, height and the length of capillary-like channels are $7.5 \mu \mathrm{m} \times 6 \mu \mathrm{m} \times 40 \mu \mathrm{m}$ and $6 \mu \mathrm{m} \times 5 \mu \mathrm{m} \times 40 \mu \mathrm{m}$ respectively, which are comparable to conventional micropipette studies.

The experimental design is shown in Fig.1D. The flow through the narrow channels will carry single cells into the trap based on the principle of flow resistance [24]. In our design, cells with the diameters of less 
than $16 \mu \mathrm{m}$ could be captured when they flow on the side of trap units. On the other hand, the geometric parameters of microfluidic chip are fine-tuned to ensure that the inequation, , is satisfied, where is the flow resistance of narrow straight channels, is the flow resistance of loop channels. Therefore, the pressure differences applied to the cells in the narrow straight channels could be kept the same whether other narrow straight channels blocked by cells or not. After the period of cell loading, around 15 minutes in our experiments, sufficient numbers of single cells could be captured. Then predefined pressures would be applied to cells for 4 hours and their dynamic behaviors would be recorded using time-lapse microscopy. In our experiments, we applied pressure differences, 50 mbar, 100 mbar, 200 mbar or 400 mbar, to the four channels in one device, corresponding to pressure drops, around $63 \mathrm{~Pa}, 125 \mathrm{~Pa}, 250 \mathrm{~Pa}$ or $500 \mathrm{~Pa}$ on captured cells. As a comparison, the blood pressure in the human body ranges from 3 $\mathrm{mmHg}$ to $120 \mathrm{mmHg}$, and the lymph pressure could be as low as $1 \mathrm{mmHg}$, that is, $133 \mathrm{~Pa}$.

To ensure the effectiveness of microfluidic device, preliminary experiments and simulations were performed to test the occupied rate of trap units and estimate the pressure drops on isolated cells. As shown in Fig.S1A, the occupied rate of trap units increases with the cell density during the period of cell loading. As illustrated in Fig. S1B, C, simulations of fluid flow using COMSOL (COMSOL Multiphysics) software indicates that as the pressure difference applied on the whole chip increases geometrically, the pressure drops exerted on single cells increase in a similar manner with an acceptable deviation from defined expected pressure drops. In our experiments, the occupied rate can be controlled at about 20-50\% using suitable cell density (Fig S1A). Under these conditions, the design of our microfluidic device could meet the experiment requirements to compare the behaviors of cells under predefined pressure differences in this work.

Under the fixed pressure, cells enter the microvessels, clog the flow, and bear the force. The cell width could fill the microvessel width in most cases to guarantee the force exerted on cells as expected. Using the microfluidic chips demonstrated previously, a large amount of data about the dynamic behaviors of single cells at fixed pressures were recorded. Customized MATLAB codes were used to identify and analyze these dynamic behaviors of the single cells across the microvessels. In our experiments, the protrusion length $L$ of single cell is defined as the length of the trailing edge of the single cell into the microvessels. Focusing on the period that cells enter the microvessels and reach the ends, two kinds of patterns of dynamic behavior were recognized through data analysis as demonstrated in Fig. 1E, F through linear fitting (see Movie S1 and S2 for details). One pattern displays excellent linearity (R-squared about 0.96 ) suggesting the Newtonian droplet state, and the other elucidates some cells might behave in a complex and nonlinear manner after adhesion with microvessels.

\section{Dynamic patterns under fixed pressure drops}

All the dynamic behaviors of cells under all different pressure drops could be observed in our experiments as depicted in Fig. 2 and Fig. S2. As described above, most of the data seems in a mess and has no obvious pattern, yet detailed analysis indicates that a portion of single cells show excellent linearity and 
the rest behave nonlinearly. In previous researches about the responses of single cells under high stresses in seconds, most results have emphasized that single cells should be considered as elastic or viscoelastic material $[5,25]$. That is, the linear elastic solid model was applied widely and worked well in this field. Nevertheless, our research demonstrates viscosity plays a dominant role instead of elasticity under low mechanic stresses for minutes to hours. A classification was performed based on R-squared of linear fitting, as demonstrated in Fig. 2. The classification threshold 0.85 was chosen to discriminate the "linear" cells from the "nonlinear" cells. Obviously, the ratio of the "linear" cell number to the number of total cells decreased from $99 \%$ to $48 \%$ when the pressure difference decreased from 400 mbar to 50 mbar. More cells exhibit complicated and nonlinear behaviors under lower pressure drop.

For most adherent cell types such as cancer cells, adhesion time scale ranges from tens of minutes to several hours [26]. And the dynamic behaviors of adherent cells could be tuned by related proteins, such as integrin, talin [2-3]. It seems to indicate that in our experiments, cells are likely to switch from the suspended state to the adherence state. The biochemical reactions during adherence and random migration might make a significant contribution to cell traverse-vessel behaviors for the "nonlinear" cases.

\section{Modified Newtonian droplet model}

Basic Newtonian droplet model regards the single cell as the Newtonian droplet [25], and the behavior of these cells could be elucidated using a linear differential equation. It means that the viscosities of these "linear" cells remain constant during the whole dynamic process. This model could only explain the linear behaviors of single cells and have no explanatory ability for the other "nonlinear" cells. As the concept shown in Fig. 3A, these "nonlinear" cells elongate in the microvessels and may adhere to the microvessels with time, then they behave more randomly.

To describe these complicated and nonlinear dynamic patterns, we illustrated the dynamic behavior mechanism as shown in Fig. 3B and modified the original Newtonian droplet model (details given in the Supplementary Information):

$$
\frac{d L}{d t}=\frac{\Delta p \cdot S-R_{f}}{6 \eta_{\text {itt }} \pi r}
$$

to\

$$
\frac{d L}{d t}=\frac{\Delta p \cdot S-R_{f}+f(t) g(L)}{6 \eta_{j u t} \pi r+\alpha A(t)}
$$


where is the protrusion length of the cell into the microvessel, is the applied pressure to the cell, is the area of the cross section of the microvessel, $R_{f}$ is the resistance corresponding to the critical excess suction pressure because of the capillary effect, $\eta_{\text {int }}$ is the intrinsic viscosity of the cell, is the equivalent radius of the cross section of the microvessel.

In the modified model, $a \cdot A(t)$ is introduced into the formula according to the cell adhere effect may greatly contribute to overcome the pressure difference, where $A(t)$ is defined as the degree of cell adhesion over time (Supplementary Fig. S3A), and $a$ is the constant coefficient between cell adhesion and additional viscosity. $f(t) \cdot g(L)$ is used to describe the active force generation during the whole traverse-vessel behavior, where $f(t)$ is the value of the active force, and $g(L)$ is tunable factor. Here, we assumed that the active forces $f(t)$ generated by cells are distributed randomly in the allowable range from $-A(t)$ to $A(t)$. And $g(L)$ depicts the effect of the protrusion length on active force generation. The simulation parameters are determined based on the previous studies (details given in the Supplementary Table S1).

As shown in Fig.3C, these simulation results are in agreement with our experiments mostly. Under low pressure drop, cells behave like Newtonian droplet before adhesion and the nonlinearity of cells become more pronounced during adhesion. Most cells behave in an excellent linear manner under high pressure drop. That is, the active force generation sourced from biochemical reactions has prominent influence on the cell behaviors during the traverse-vessel process. In order to confirm our conjectures, this parameterized model was used to simulate cell behaviors in narrower microvessels. As expected, Fig. 3D illustrates that more cells behave "nonlinear" in narrower microvessels under the same pressure drop. Detailed data analyses demonstrated that our model could explain most experimental phenomena for two sizes of microvessels (Supplementary Fig. S4, Fig. S5).

\section{Distribution analysis of cells' behaviors illustrated mode switch}

As discussed in the model and equations (1) and (2), the cells may act like Newton droplet phase and transform to adhesion/migration phase with the increase of contact time. Besides R-squared, we also used apparent viscosity $\left(\eta_{a p p}\right)$ to indicate the cell behavior changes. The apparent viscosity $\left(\eta_{a p p}\right)$ is defined as:

$$
\eta_{c p p}=\frac{\Delta p \cdot S-R_{f}}{6 \pi \cdot E^{\&}}
$$

As shown in Fig. 4A, under the constant pressure drop, the major peak (b/ue) shifts towards higher apparent viscosity indicating the apparent viscosity of cells gradually rises. Meanwhile, a weak peak (pink) at greater apparent viscosity occurs and increases in the proportion of total over time. This behavior is more remarkable under the lower constant pressure drop, while under the higher pressure drop cells often traverse through the capillary before they could adhere to their surrounding environment. The R-squared distribution in Fig. 4B could also offer support for this conjecture. Under the lower consistent pressure drop, cells mostly show a relatively great linear behavior within dozens of minutes, then cells 
progressively make the transitions from Newtonian droplet state to adhesion/migration state. Finally, cells completed the transitions and exhibited non-linear behaviors. All the distributions of apparent viscosity and R-squared within four hours can be found in the Supplementary Fig. S6 (experiment) and Fig. S7 (simulation).

To further confirm the transition and compare experiments with theories, the numbers of cells with specific R-squared and apparent viscosities in experiments and simulations are drawn in Fig. 5A, B. It is worth noting that Fig. 5A counts cells between 20 and 40 minutes and Fig. 5B counts cells between 40 and 80 minutes both under the pressure difference 50 mbar. These results indicate a negative correlation between the apparent viscosities of single cells and R-squared regardless of in experiments or simulations. Besides, the heatmaps also show two evident clusters in the upper left and the lower right suggesting the existence of two potential distinct states for cells. One state with lower apparent viscosities and higher R-squared close to 1 , and the other with higher apparent viscosities and lower Rsquared close to 0 . To eliminate the doubt about whether the number of single cells is enough for proving this conjecture, a heatmap depicting the accumulation of the numbers of cells during different periods is shown in Fig. S8. And the comparison of Fig. S8A and Fig. S8F could elucidate the transition between these two states over time. Similar results are also observed in simulations as Fig. S9 shown. Taken together, it is assured that cancer cells could switch their state from Newtonian droplet state to adhesion/migration state.

Throughout these transitions, apparent viscosities of cells increased initially and then leveled off with time indicating cells attaches to the surrounding environments completely as shown in Fig. 5C. Meanwhile, Fig. 5D suggests that the dynamic behaviors of cells are influenced by cell adhesion and become more nonlinear than before. Finally, considering the residual cells before traversing the microvessels, the ratio of these cells to total implies two potential distinct stages, a rapid decline at the initial stage and a steady decline at the latter stage, as plotted in Fig. 5E using dotted lines, which also suggests the mode switch. From the beginning to the end, the biochemical reactions have little effect initially while dominate these transitions finally. Experiments and simulations both verified these phenomena qualitatively, although they might not match perfectly from a view of quantitative because elapsed time of cell loading cannot be guaranteed to be the same in all experiments and parameters chosen in simulations might not be best suited for all experiments.

\section{Further discussions for pressures and vessel sizes effect for the cell behaviors}

The dynamic behavior of cells in the microvessels with different sizes under different pressure applied is summarized and compared in Fig. 6 to verify our parameterized model further.

In order to compare the R-squared and the apparent cell viscosity of the cell traverse-vessel behaviors at different conditions in experiments with simulations, simulations were performed densely under the pressure drop which ranged from $63 \mathrm{~Pa}$ to $500 \mathrm{~Pa}$. Fig. $6 \mathrm{~A}$ shows that the median value of the fitted Rsquared of all cell behaviors with different pressure drops. With the pressure drops decreased, the Rsquared decreased from about 1.0 to a pretty low value, $\sim 0.8$ for microvessels with $7.5 \mu \mathrm{m} \times 6 \mu \mathrm{m} \times 40 \mu \mathrm{m}$ 
and $\sim 0.4$ for microvessels with $6 \mu \mathrm{m} \times 5 \mu \mathrm{m} \times 40 \mu \mathrm{m}$. The smaller the microvessel size was, the larger decrease of the median value of R-squared would be. In contrast, Fig. 6B demonstrates the median of the apparent viscosities $\eta_{a p p}$ of cells increase greatly from $\sim 3000 \mathrm{~Pa} \cdot \mathrm{s}$ as the applied pressure decreased. The value of cell apparent viscosities matches with previous studies approximately [27-29]. Experiments are in good agreement with simulation results in Fig. 6A, B.

To illustrate the core concept, the schematics of two kinds of dynamic patterns are depicted as shown in Fig. 6C. Before adhesion, cells could linearly traverse the capillary-like vessels and their apparent viscosities $\eta_{a p p}$ are approximate to real viscosities $\eta_{\text {int }}$ of these cells. On the other hand, if pressure is too low or the microvessel is much smaller, the cells would adhere to the microvessels and generate active force to speed up or slow down their migration. After adhesion, these cells behave randomly and nonlinearly, then the apparent viscosities $\eta_{a p p}$ of these cells are much larger than their real viscosities $\eta_{\text {int }}$. In this state, these cells could maintain their physiological conditions and seed in current sites, which means that cells might become more aggressive and form a metastatic tumor. In other words, if we could reduce the viscosities of cancer cells or the adhering force between cancer cells and the microvessels, the cancer metastasis might be restrained to some degree.

\section{Summary And Discussion}

We designed a high-throughput microfluidic system with external pneumatic pump to research the dynamic behaviors of cancer cells at long timescales responding to low mechanical stresses. We have demonstrated that cancer cells might behave in two quite different patterns, "linear" and "nonlinear", when they are exerted low pressure drop on, and this transition from "linear" to "nonlinear" is continuous as the applied pressure decreases from $500 \mathrm{~Pa}$ to $63 \mathrm{~Pa}$. Using the R-squared of linear fitting as the evaluation criterion, the ratio of the "nonlinear" cell number to the number of total cells increases during the reduction of applied pressure. To explain these phenomena, we modified the Newtonian droplet model and introduced the influence of biochemical reactions which happens in cell adhesion and random migration. Besides, simulations and experiments could match well regardless of different sizes of microvessels and different applied pressures. Generally speaking, we verified that how the size of vessels and the pressure differences, affected cancer cells to seed and migrate at distant organ sites. We believe that our research could provide some useful insights for cancer metastasis and cancer therapy.

\section{Materials And Methods}

Cell culture. Metastatic human breast cancer (MCF-7) used in the test was purchased from America Type Culture Collection(ATCC). Cells were cultured in Dulbecco's Modified Eagle Medium (DMEM; Gibco) supplemented with $10 \%$ fetal bovine serum (FBS; Hyclone), $100 \mathrm{U} / \mathrm{mL}$ penicillin and streptomycin (Gibco) at $37^{\circ} \mathrm{C}$ in $5 \% \mathrm{CO}_{2}$.

Device fabrication. Photolithography technique was used to fabricate the master molds. Briefly, the mold 1 of layer one for cell loading and pressure operation was fabricated by SU8 (MicroChem, USA) with two 
heights. Then, the mixture ( $\mathrm{A}: \mathrm{B}=8: 1)$ of prepolymer polydimethylsiloxane (PDMS) was spin-coated on the mold of layer one with a height of about $50 \mu \mathrm{m}$ and cross-linked at $70{ }^{\circ} \mathrm{C}$ for 2 hours. The mold 2 of layer two for $5 \% \mathrm{CO}_{2}$ supply was also fabricated by the SU8 with the height of $40 \mu \mathrm{m}$. A mixture $(\mathrm{A}: \mathrm{B}=12: 1)$ of PDMS was cast on the mold of layer two with height about $8 \mathrm{~mm}$ and also cross-linked at $70{ }^{\circ} \mathrm{C}$ for 2 hours. Then the PDMS layer on the mold 2 was peeled off and bonded to the layer one by air plasma as shown in Fig. 1A. After peeled off the bonded PDMS piece from mold 1 and punched the inlets and outlets, the PDMS piece bonded to the glass slide by air plasma. Once the PDMS chip was sealed against the glass slide, the device was placed in an oven at $70^{\circ} \mathrm{C}$ overnight to improve bonding quality before use.

Experiments and analysis. Before the experiments, the device was exposed to UV and degassed in vacuum for $30 \mathrm{~min}$ in order to sterilize. We used $0.25 \%$ Trypsin-EDTA solution to dissociate MCF-7 cells at $37^{\circ} \mathrm{C}$ for $3 \mathrm{~min}$. These cells were resuspended to a concentration of $10^{5} / \mathrm{mL}$ in normal media. Cells were loaded into the device through the inlets and trapped into the capillary shaped microvessels for 15 min under the fixed pressure (50 mbar). Then, the sample positions were picked up under a Nikon Ti microscope with a standard dry objective (Nikon LWD 40×/0.55 Ph2 ADL) after stopping the air-pressure pump to ensure the recovery of trapped cells (about $10 \mathrm{~min}$ ). After setting different pressures for different channels by a four-channel pressure operator (Fluidic controller, MesoBiosystem, China), the microscope system captured images at 2 min per frame in the regions of interest. Zoom knob was set to $1.5 \times$ for more magnification. Experiments typically lasted 4 hours. Experimental analysis and cell tracking were performed using custom MATLAB programs.

\section{Declarations}

Code availability. The MATLAB codes in the current study are available from the corresponding author on reasonable request.

Data availability. All data generated or analyzed during this study are included in this article and its supplementary information files. These datasets are also available from the corresponding author on reasonable request.

\section{Author Information}

\section{Corresponding Authors}

*E-mail: pkuluocx@pku.edu.cn.

\section{Author Contributions}

X.L. and C.L. designed research; X.L., J.S., Z.G., J.X. and S.W. performed the experiments; X.L., J.S., C.L. and Q.O. analyzed data; X.L. and C.L built the model; X.L. performed the simulations; and X.L. and C.L. wrote the paper. 


\section{Competing Interest Statement}

The authors declare no conflict of interest.

\section{Notes}

The authors declare no competing financial interest.

\section{Acknowledgments}

We would like to thank Yugang Wang, Gen Yang, Feng Liu for helpful discussions. This study was supported by the NSFC of China (11974002, 11774011 and 12094054) and the National Key Research and Development Project (SQ2018YFA090070-03 and 2020YFA0906900).

\section{References}

1. Massagué, J. \& Obenauf, A. C. Metastatic colonization by circulating tumour cells. Nature $\mathbf{5 2 9 , 2 9 8 -}$ 306 (2016).

2. Devreotes, P. \& Horwitz, A. R. Signaling networks that regulate cell migration. Cold Spring Harbor Perspectives in Biology 7, (2015).

3. Ridley, A. J. Cell migration: Integrating signals from front to back. Science 302, 1704-1709 (2003).

4. Wu, P.-H. et al. A comparison of methods to assess cell mechanical properties. Nature Methods 15, 491-498 (2018).

5. Rodriguez, M. L., McGarry, P. J. \& Sniadecki, N. J. Review on cell mechanics: Experimental and modeling approaches. Applied Mechanics Reviews 65, (2013).

6. Hogan, B., Babataheri, A., Hwang, Y., Barakat, A. I. \& Husson, J. Characterizing cell adhesion by using micropipette aspiration. Biophysical Journal 109, 209-219 (2015).

7. Houk, A. R. et al. Membrane tension maintains cell polarity by confining signals to the leading edge during neutrophil migration. Cell 148, 175-188 (2012).

8. Mak, M. \& Erickson, D. A serial micropipette microfluidic device with applications to cancer cell repeated deformation studies. Integrative Biology 5, 1374-1384 (2013).

9. Lee, L. M. \& Liu, A. P. A microfluidic pipette array for mechanophenotyping of cancer cells and mechanical gating of mechanosensitive channels. Lab on a Chip 15, 264-273 (2015).

10. Lange, J. R. et al. Unbiased high-precision CELL Mechanical measurements with microconstrictions. Biophysical Journal 112, 1472-1480 (2017).

11. Gossett, D. R. et al. Hydrodynamic stretching of single cells for large population mechanical phenotyping. Proceedings of the National Academy of Sciences 109, 7630-7635 (2012).

12. Chen, Z. et al. Cell elasticity measurement using a microfluidic device with real-time pressure feedback. Lab on a Chip 20, 2343-2353 (2020). 
13. Au, S. H. et al. Clusters of circulating tumor cells traverse capillary-sized vessels. Proceedings of the National Academy of Sciences 113, 4947-4952 (2016).

14. Luo, T., Mohan, K., Iglesias, P. A. \& Robinson, D. N. Molecular mechanisms of cellular mechanosensing. Nature Materials 12, 1064-1071 (2013).

15. Paul, C., Mistriotis, P. \& Konstantopoulos, K. Cancer cell motility: lessons from migration in confined spaces. Nature Reviews Cancer 17, 131-140 (2016).

16. Liu, Y. et al. Confinement and Low Adhesion Induce Fast Amoeboid Migration of Slow Mesenchymal Cells. Cell 160, 659-672 (2015).

17. Holle, A. et al. Cancer Cells Invade Confined Microchannels via a Self-Directed Mesenchymal-toAmoeboid Transition. Nano Letters 19, 2280-2290 (2019).

18. Wang, M. et al. Microchannel Stiffness and Confinement Jointly Induce the Mesenchymal-Amoeboid Transition of Cancer Cell Migration. Nano Letters 19, 5949-5958 (2019).

19. Zhao, R. et al. Hydraulic resistance induces cell phenotypic transition in confinement. Science Advances 7, eabg4934 (2021).

20. Williams, S. et al. Dynamic measurement of human capillary blood pressure. Clinical Science 74, 507-512 (1988).

21. Sloas, D. et al. Estimation of the Pressure Drop Required for Lymph Flow through Initial Lymphatic Networks. Lymphatic Research and Biology 14, 62-69 (2016).

22. Pereira, E. et al. Lymph node metastases can invade local blood vessels, exit the node, and colonize distant organs in mice. Science 359, 1403-1407 (2018).

23. Headley, M. et al. Visualization of immediate immune responses to pioneer metastatic cells in the lung. Nature 531, 513-517 (2016).

24. Tan, W. \& Takeuchi, S. A trap-and-release integrated microfluidic system for dynamic microarray applications. Proceedings of the National Academy of Sciences 104, 1146-1151 (2007).

25. Lim, C., Zhou, E. \& Quek, S. Mechanical models for living cells-a review. Journal of Biomechanics 39, 195-216 (2006).

26. Schlie, S., Gruene, M., Dittmar, H. \& Chichkov, B. Dynamics of Cell Attachment: Adhesion Time and Force. Tissue Engineering Part C: Methods 18, 688-696 (2012).

27. Hu, S. \& Lam, R. Characterization of viscoelastic properties of normal and cancerous human breast cells using a confining microchannel. Microfluidics and Nanofluidics 21, (2017).

28. Plaza, G. et al. Simple measurement of the apparent viscosity of a cell from only one picture: Application to cardiac stem cells. Physical Review E 90, (2014).

29. Yuan, W., Xiang, C., Xue, C., Liu, B. \& Qin, K. Measuring the apparent viscosities of single cells by tracking the entire deformation dynamics in microfluidic channels. Analytical Methods 11, 56805690 (2019).

\section{Figures}


A)

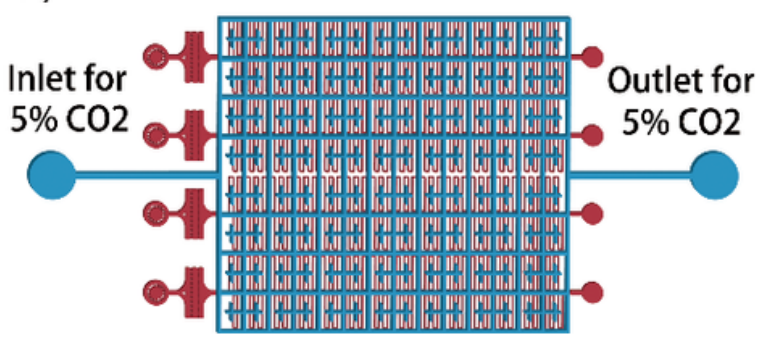

B)

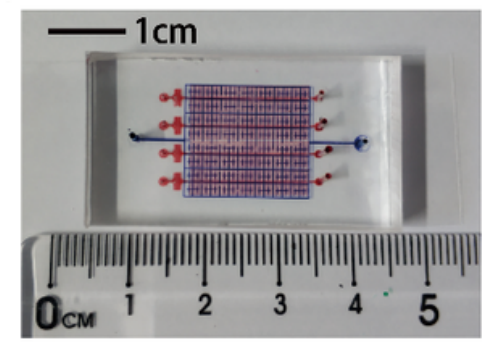

C)

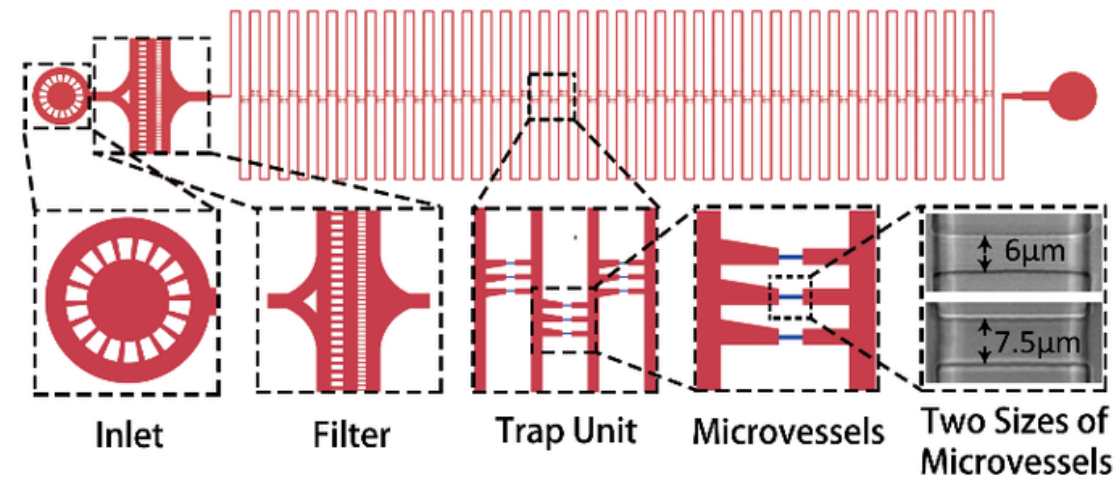

E)
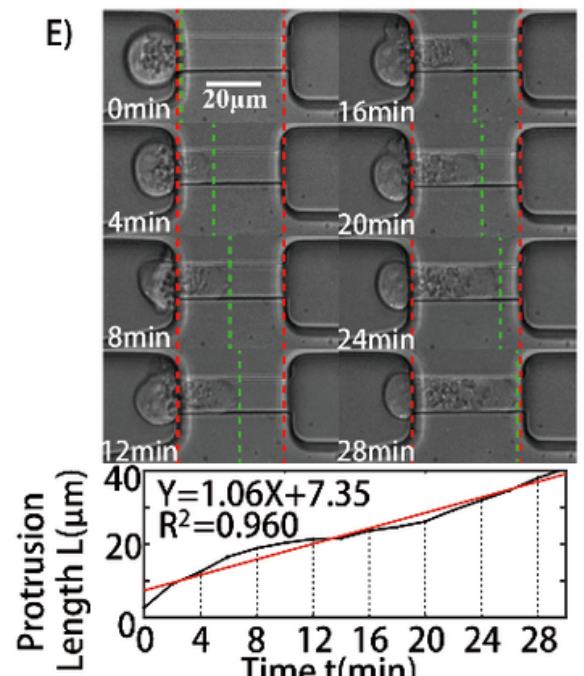

$Y=1.06 X+7.35$
$R^{2}=0.960$

20 .

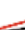

?

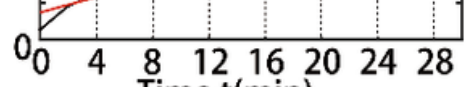

F)

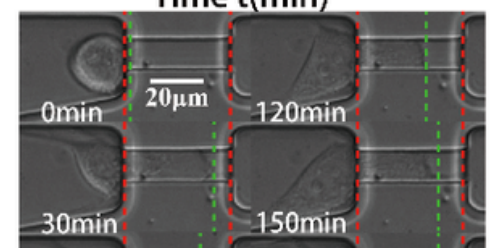

D) Cell Loading(15min)

Pressure Applied to Cells(4h)
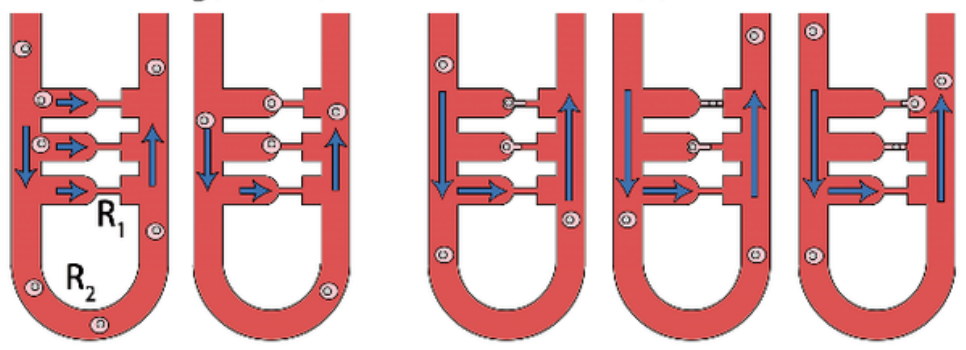

(2) Cancer Cell

$\Rightarrow$ Flow

Flow Resistance

$R_{1}>10 \times R_{2}$

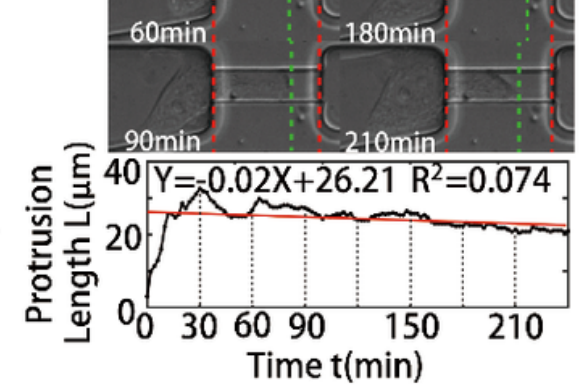

Figure 1

The design principle of the microfluidic device and time-lapse microscopy images of two typical cells with different traverse-vessel behaviors. (A) Schematic diagram of the double-layer microfluidic chip with CO2 layer (blue) and cell cultivation layer (red) to maintain cell culture environment and culture cells. (B) A photograph of the microfluidic chip with $\mathrm{CO} 2$ layer (upper, blue) and cell cultivation layer (bottom, red). Scale bar: $1 \mathrm{~cm}$. (C) Detailed schematic diagrams of the cell cultivation layer with Inlet, Filter, Trap Unit and Outlet. (D) Schematic illustration of experimental design in the research. (E) (F) Time-lapse microscopy images and the protrusion length as a function of time for two typical cells under applied pressure difference $\Delta " P^{\prime \prime}, 200$ mbar and 100 mbar respectively. Scale bar: $20 \mu \mathrm{m}$. 

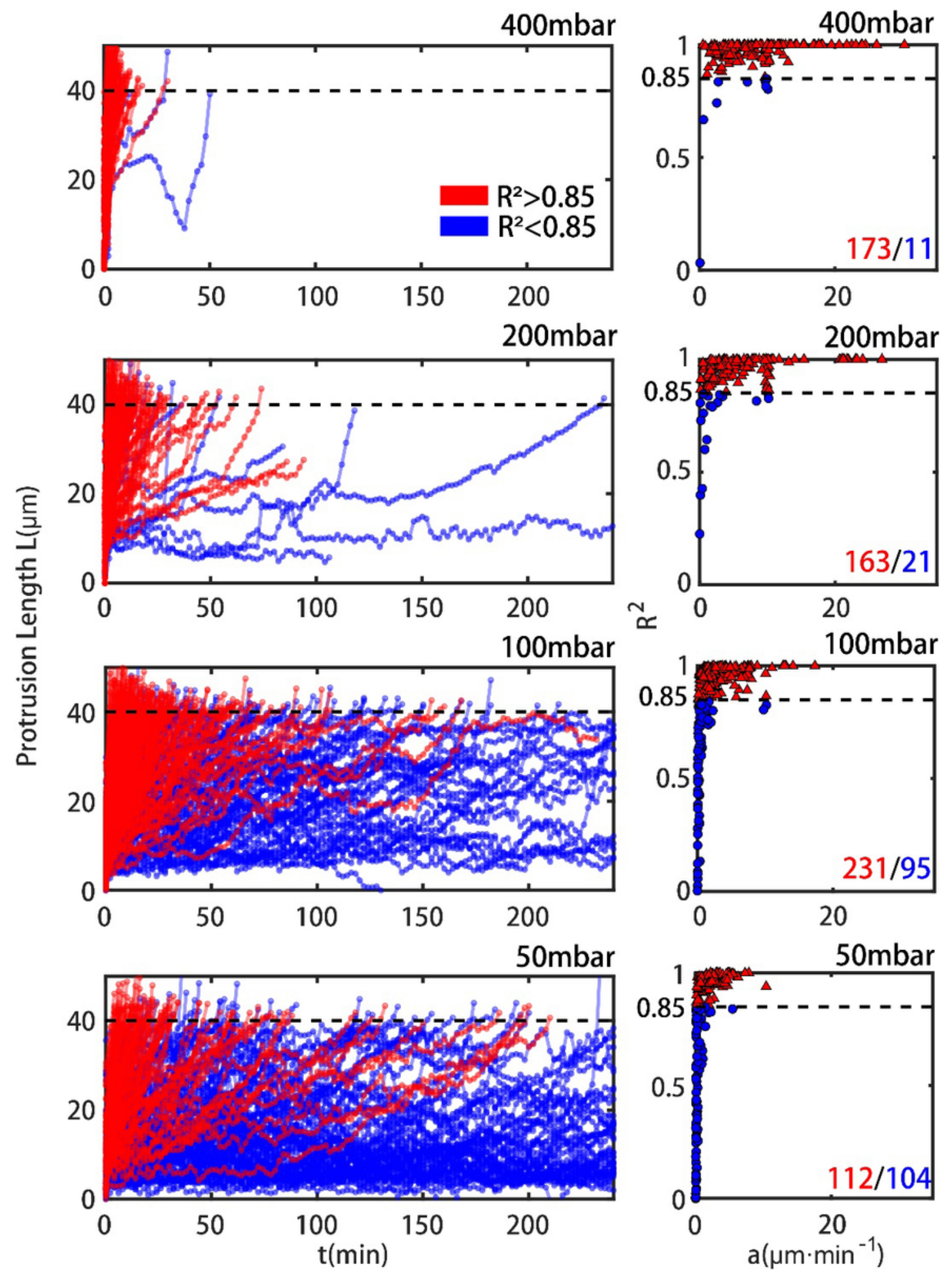

Figure 2

Left: Dynamic behaviors of cancer cells traversing microvessels with $7.5 \mu \mathrm{m} \times 6 \mu \mathrm{m} \times 40 \mu \mathrm{m}$ over time under four applied pressure differences $\Delta " P^{\prime \prime}$ (400 mbar, 200 mbar, 100 mbar and 50 mbar); Right: Scatter plot of R-square and fitting velocities for cells traversing microvessels with $7.5 \mu \mathrm{m} \times 6 \mu \mathrm{m} \times 40 \mu \mathrm{m}$ under four applied pressure differences $\Delta^{\prime} \mathrm{P}^{\prime \prime}$ (400 mbar, $200 \mathrm{mbar}, 100 \mathrm{mbar}$ and $50 \mathrm{mbar}$ ). Red lines and Blue lines indicated cells with R-squared of linear fitting larger or smaller than 0.85 . 
A)

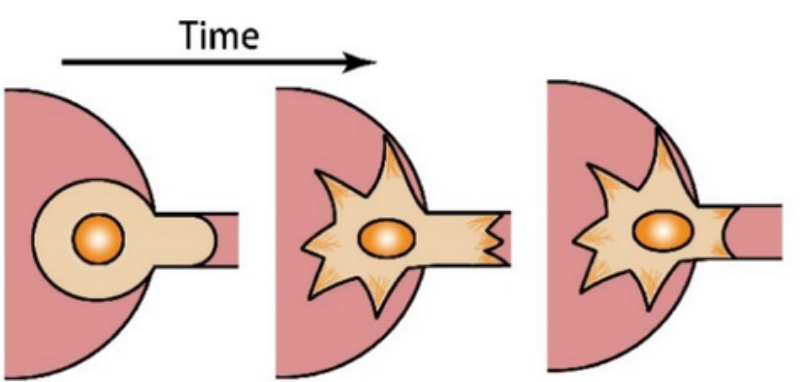

Elongation

Adhesion

Random Migration

B)

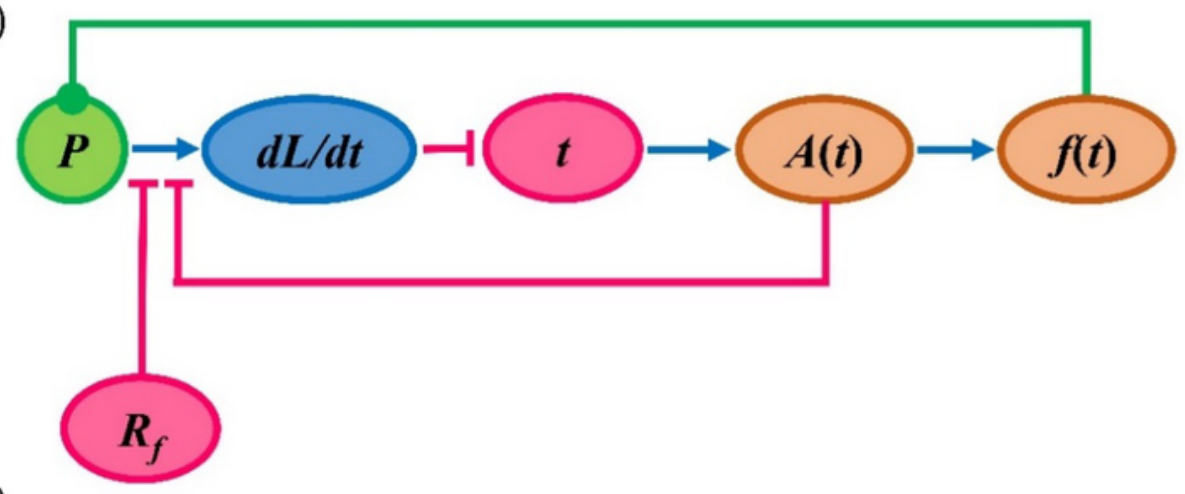

C)
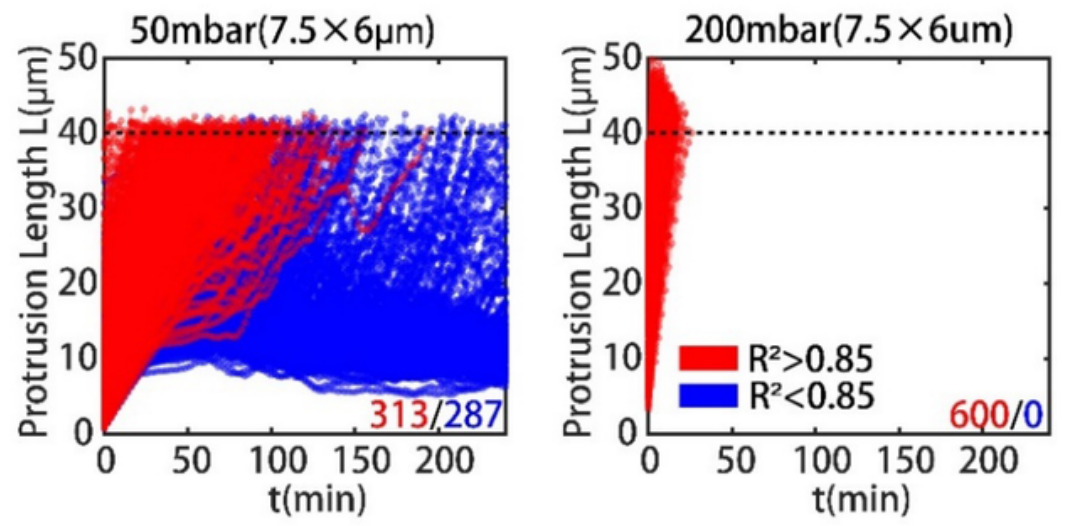

D)
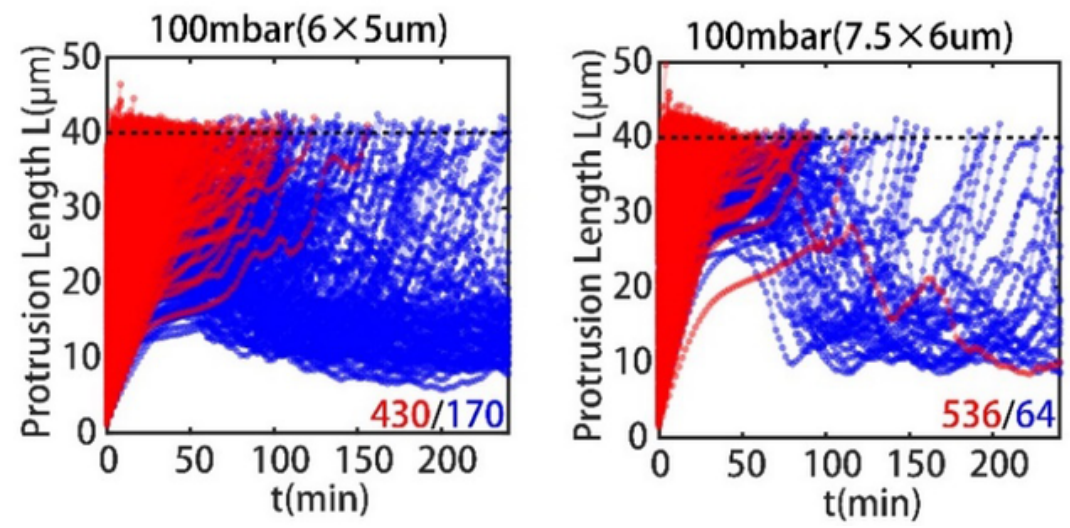

\section{Figure 3}

The model and the simulation of traverse-vessel behaviors. (A) Sketch map of cell elongate, and adhere/migration in the microvessel. (B) The model for the dynamic behavior of cells in the microvessel. (C) The simulation of dynamic behaviors for cells under different pressure differences (50 mbar and 200 mbar) using the microfluidic chips with the same size of microvessels $(7.5 \mu \mathrm{m} \times 6 \mu \mathrm{m})$. (D) The simulation 
of dynamic behaviors for cells under the same pressure difference (100 mbar) using the microfluidic chips with different sizes of microvessels $(6 \mu \mathrm{m} \times 5 \mu \mathrm{m}$ and $7.5 \mu \mathrm{m} \times 6 \mu \mathrm{m})$.

A)

Pressure (mbar)
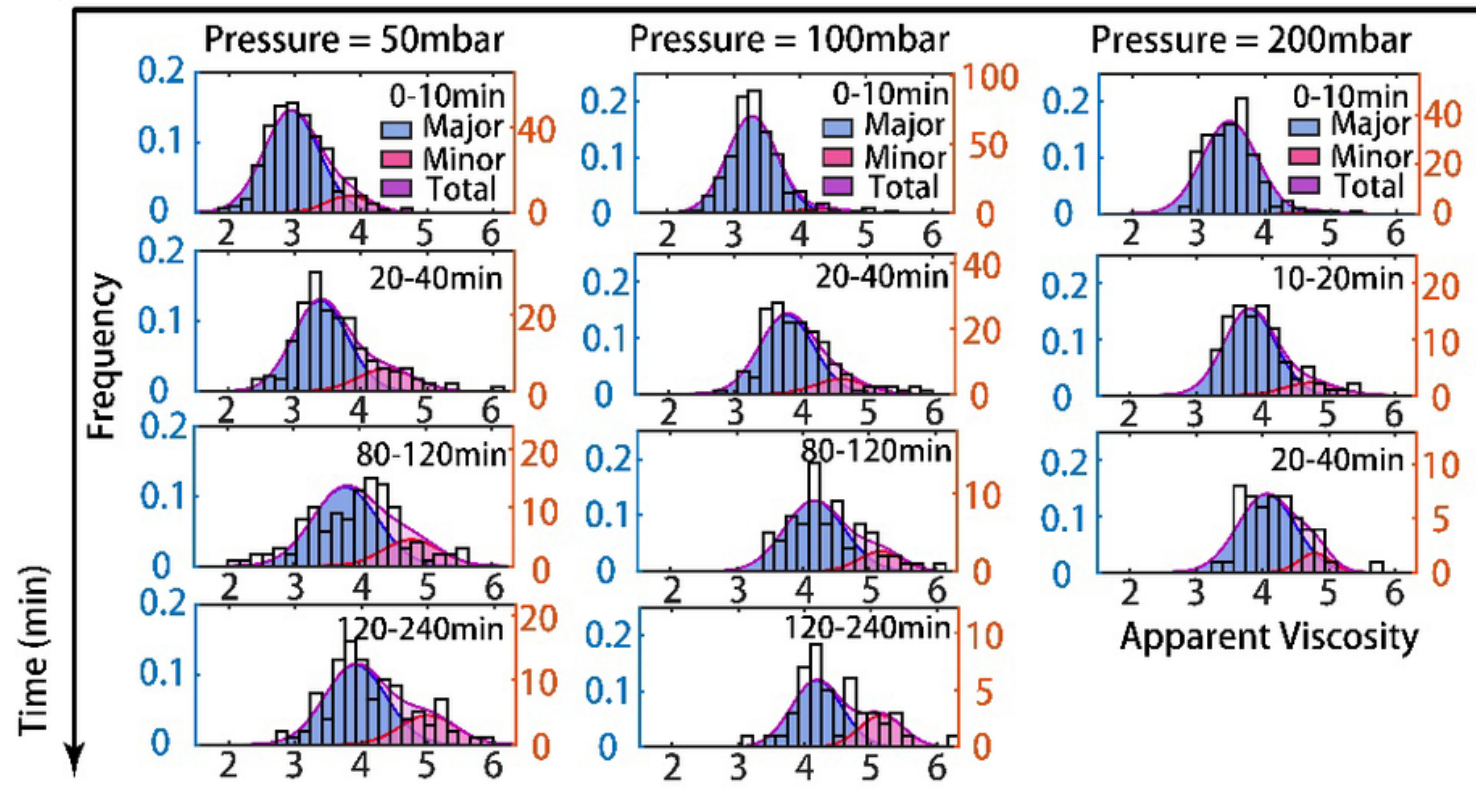

Pressure $=400 \mathrm{mbar}$
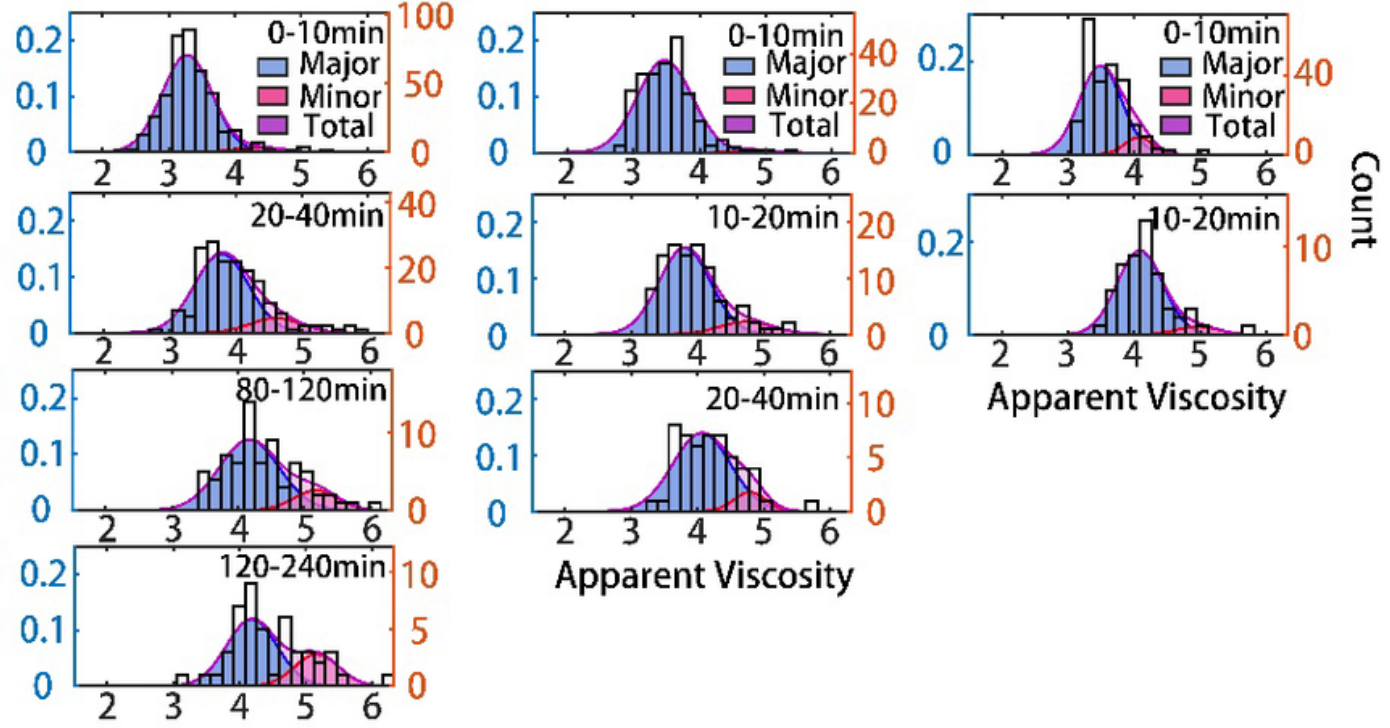

Apparent Viscosity $\left(\log _{10}(\mathrm{~Pa} \cdot \mathrm{s})\right) \quad$ Apparent Viscosity

B)

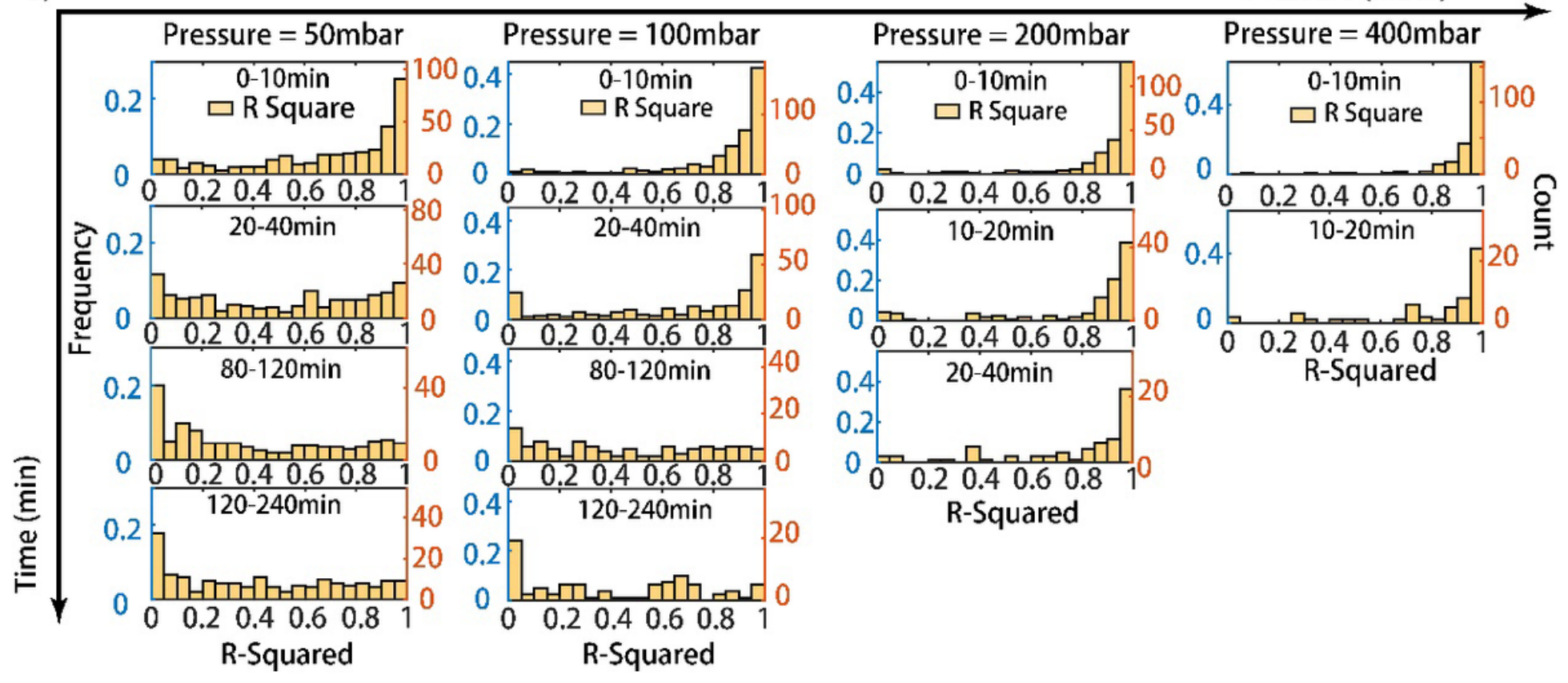

Figure 4

Distribution analysis of cells' behaviors illustrated the transition (A) Probability density distribution of cell apparent viscosities for 50, 100, and 200 and 400 mbar at various periods (0-10min, 20-40min, 80120min, 120-240min). Major peak in blue, minor peak in pink and total in purple. (B) Probability density distribution of the R-squared of linear fitting for 50, 100, and 200 and 400 mbar at various times. The left $y$-axis shows frequencies and the right $y$-axis gives counts for both (A) and (B). 
A)
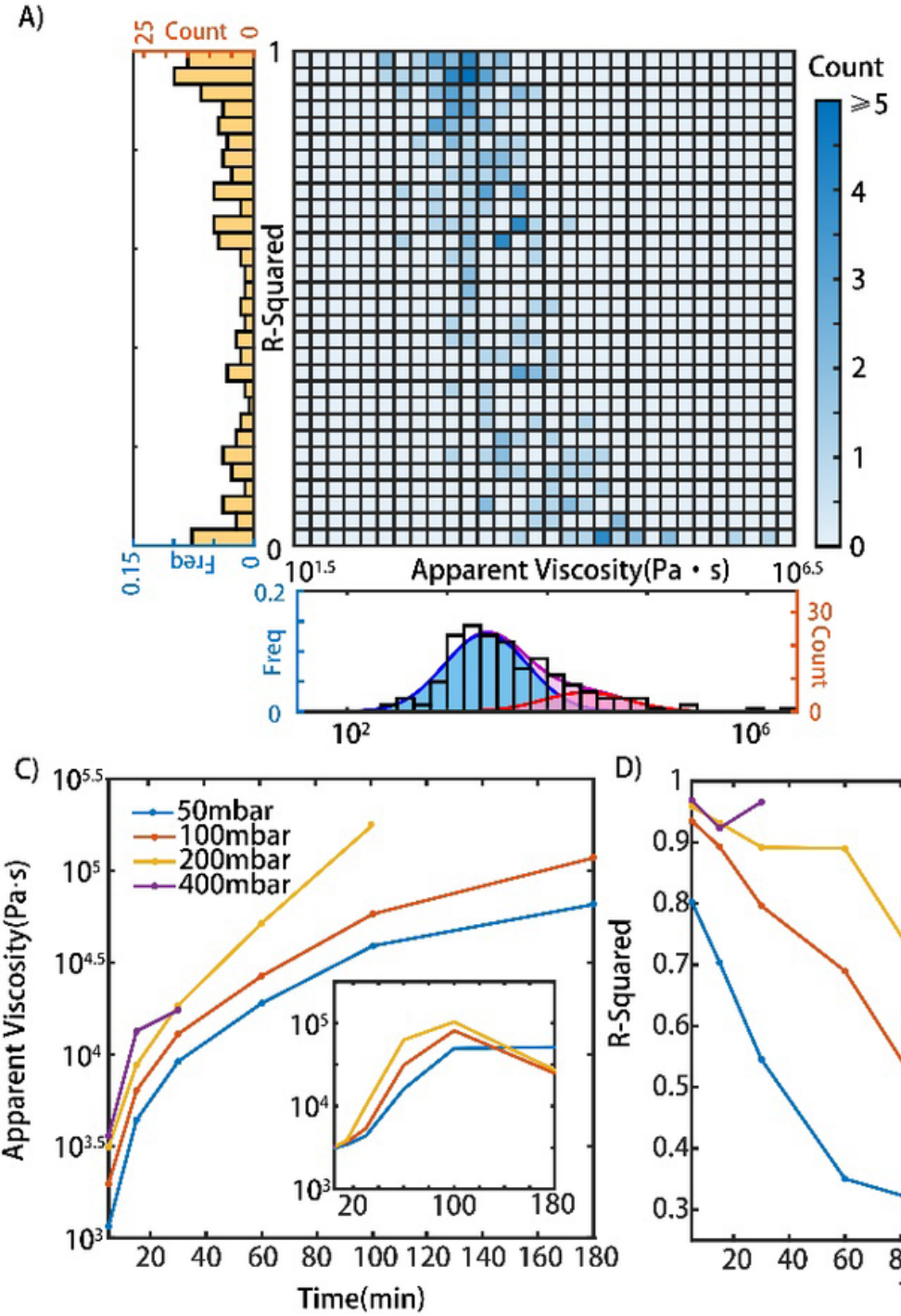

B)

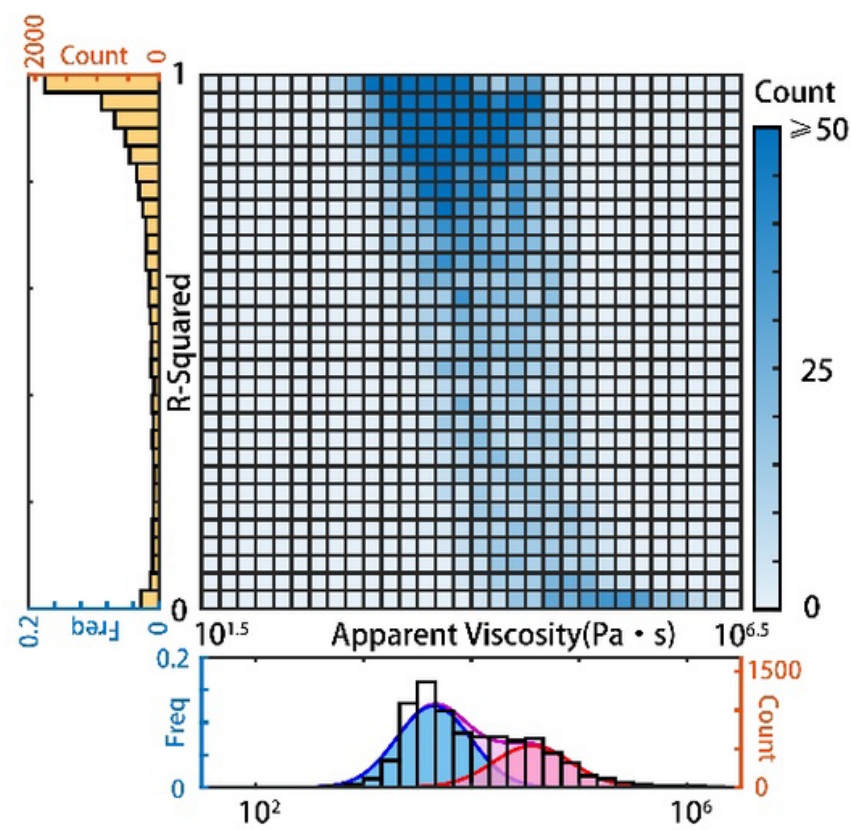

D) 1

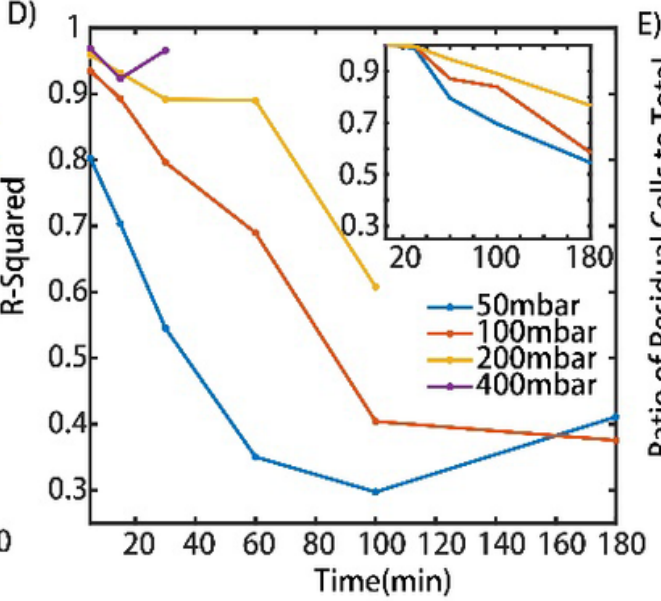

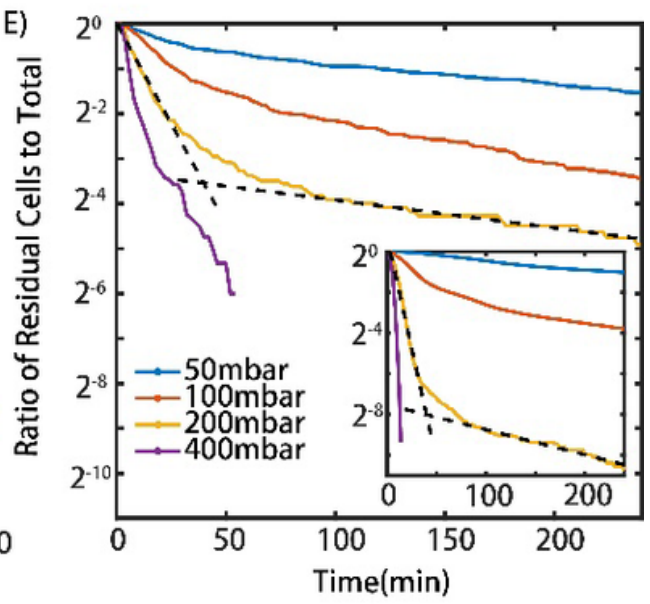

Figure 5

(A)(B) Heatmap depicting the number of cells with corresponding R-squared and apparent viscosities under the pressure difference 50 mbar. A: Counts cells between 20 and 40 minutes in experiments. B: Counts cells between 40 and 80 minutes in simulations. (C) (D) Medians of apparent viscosities and Rsquared of cells (up to 400 cells in the first time frame under the pressure difference 50 mbar, no less than 20 cells in the final time frame under the pressure difference 400 mbar) over time under different pressure differences in experiments. Insets are in simulations. (E) Ratio of residual cells (larger than 200 cells in any condition at the start of experiments) to total throughout the experiments under different pressure differences. Inset is in simulations. Dashed lines represent results of linear fitting for two distinct stages. 
A)

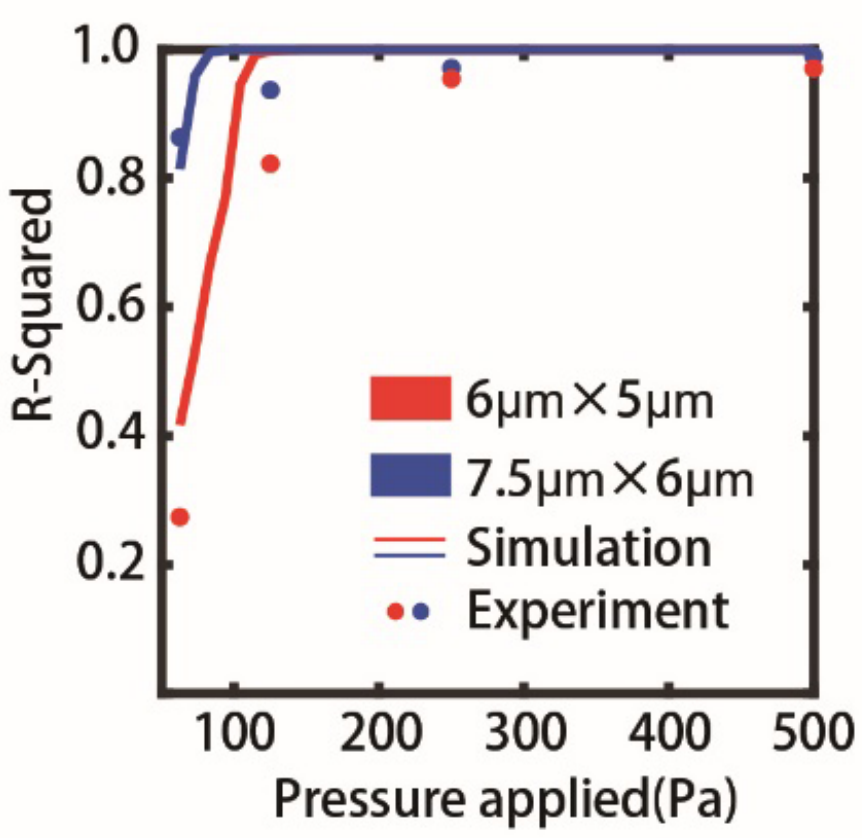

C)

$$
R^{2} \sim 1
$$

Apparent viscosity $\eta_{\text {app }} \sim \eta_{\text {int }}$
B)

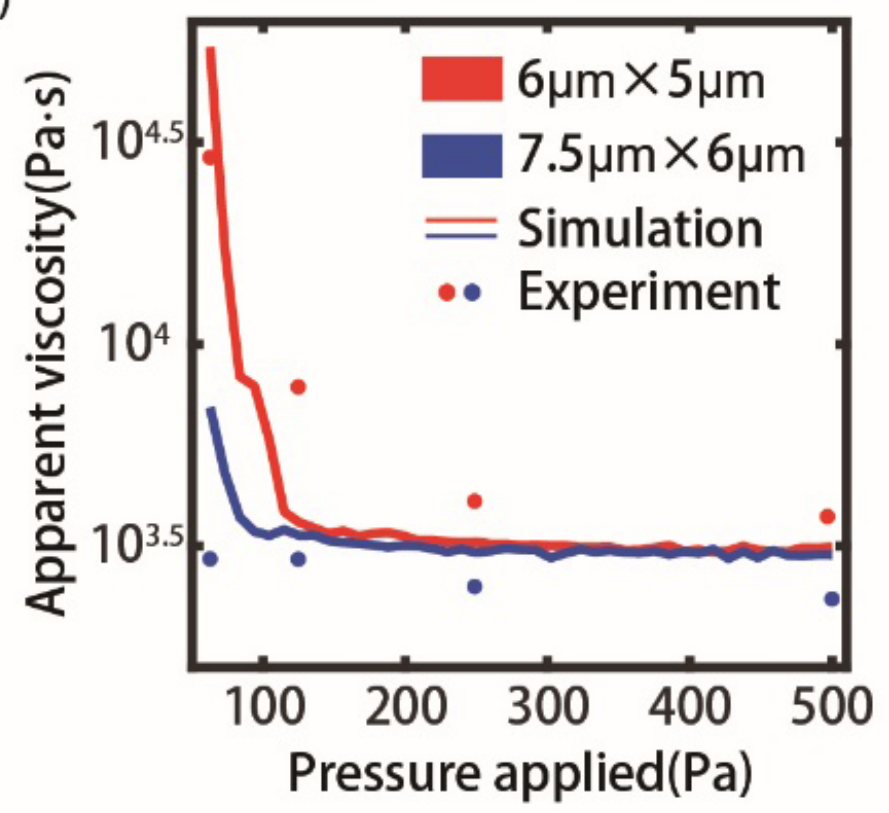

$R^{2} \sim 0$

Apparent viscosity $\eta_{a p p} \gg \eta_{\text {int }}$

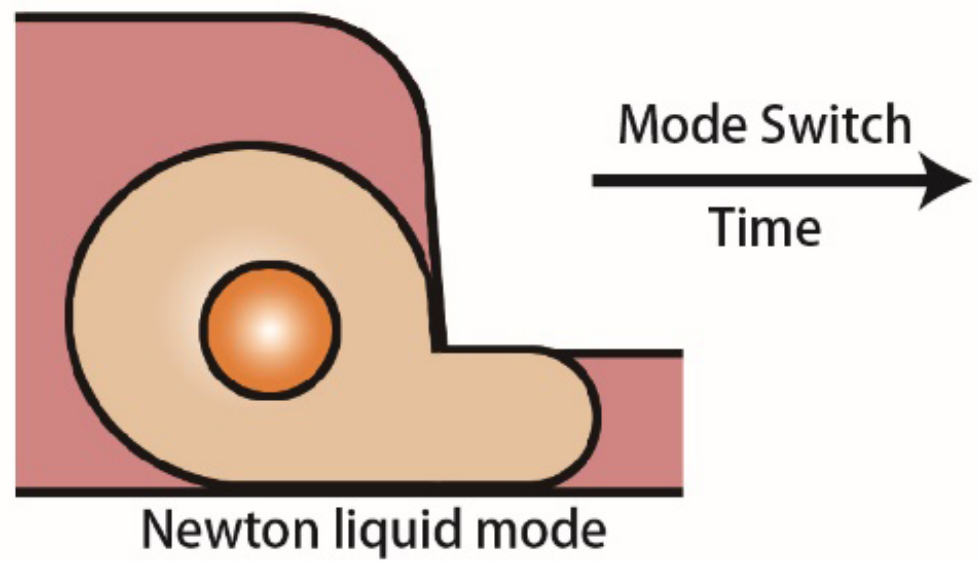

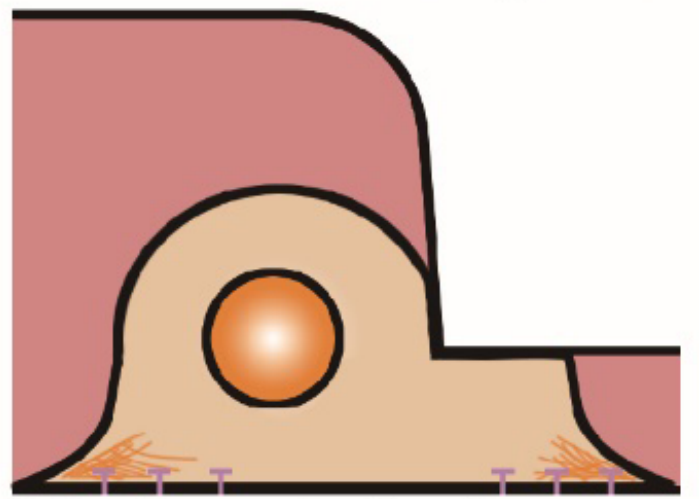

Adhesion and migration mode

Figure 6

The summarization of the R-squared and the apparent cell viscosity of the cell traverse-vessel behaviors at different conditions. (A)(B) The comparison of plots of R-squared and cell apparent viscosity as functions of pressure applied for experiments and simulations under different pressure differences (median of all available cells in one condition, and no less than 80 cells in any condition at any time). (C) Illustration of cell transition from Newtonian liquid mode to adhesion and migration mode.

\section{Supplementary Files}

This is a list of supplementary files associated with this preprint. Click to download. 
- MovieS2.mov

- SI20210826.docx

Page 18/18 